Revue internationale P.M.E.

Économie et gestion de la petite et moyenne entreprise

\title{
PME et technologies de l'information : de la prise de décision à la mise en œuvre
}

\section{Marie-Christine Monnoyer-Longé}

Volume 15, numéro 3-4, 2002

URI : https://id.erudit.org/iderudit/1008811ar

DOI : https://doi.org/10.7202/1008811ar

Aller au sommaire du numéro

Éditeur(s)

Presses de l’Université du Québec

ISSN

0776-5436 (imprimé)

1918-9699 (numérique)

Découvrir la revue

Citer cet article

Monnoyer-Longé, M.-C. (2002). PME et technologies de l'information : de la prise de décision à la mise en œuvre. Revue internationale P.M.E., 15(3-4),

11-36. https://doi.org/10.7202/1008811ar
Résumé de l'article

Le caractère transversal des TIC par rapport aux technologies spécialement conçues pour des processus productifs ciblés rend leur introduction plus complexe dans des entreprises de taille réduite. Pour appréhender les difficultés des dirigeants et de leur encadrement, nous avons analysé le cheminement de la prise de décision et de l'application de ces technologies dans 20 entreprises. Le rôle initiateur du dirigeant et de son environnement externe ont été mis en évidence, comme celui de la proactivité de toute l'équipe. Une classification des entreprises est proposée qui rend compte du poids relatif des différentes influences. 


\title{
PME et technologies de l'information: de la prise de décision à la mise en œuvre
}

Marie-Christine MONNOYER-LONGÉ

Université de Toulouse I

Université Montesquieu, Bordeaux IV

\author{
MOTS CLÉS
}

PME - TIC - Décision d'implantation - Usages

\begin{abstract}
RÉSUMÉ
Le caractère transversal des TIC par rapport aux technologies spécialement conçues pour des processus productifs ciblés rend leur introduction plus complexe dans des entreprises de taille réduite. Pour appréhender les difficultés des dirigeants et de leur encadrement, nous avons analysé le cheminement de la prise de décision et de l'application de ces technologies dans 20 entreprises. Le rôle initiateur du dirigeant et de son environnement externe ont été mis en évidence, comme celui de la proactivité de toute l'équipe. Une classification des entreprises est proposée qui rend compte du poids relatif des différentes influences.
\end{abstract}

\section{ABTRACT}

Using IT in SMF is far more complex than using any other industrial technology. Managers who are often specialists are not so frequently aware of the IT possibilities. To understand these managers' difficulties, we have analysed how the decision to invest and to implement is taken. We have interviewed 20 firms and met many of their executives. The top manager and his personal network has a very decisive

\section{AUTEURE}

Marie-Christine Monnoyer-Longé est professeure en sciences de gestion, à l'Université de Toulouse I. Elle est également coresponsable de l'équipe Lerass et présidente du Réseau européen Services Espace (RESER). Elle a en outre mené des travaux sur les usages des TIC et le développement du commerce électronique. Adresse : Université Toulouse I, place Anatole-France, 31042 Toulouse cedex, Lerass, UPS. Courriel : marie.christine.monnoyer @univ-tlse1.fr 
role, but the pro-activity of the whole team is determinant for the generalization of the IT uses in the firm. To show the role of each influence, we propose a typology of the different situations.

\section{RESUMEN}

Las TIC siendo màs transversales que las tecnologias concebidas especialmente para procesos productivos precisos, su introduccion en empresas medianas es mas complejo. Para aprender las dificultades que tienen los dirigentes y sus ejecutivos, hemos estudiado en nuestra analisis sus mecanismos de tome de decision e implementacion. Nos aparecio que los dirigentes y sus entornos externos son aun mas iniciadores de las decisiones que todos sus equipes son pro activos. Proporemos una primera clasificacion de las empresas tomando en cuenta la importancia relativa de varias influencias.

\section{ZUSAMMENFASSUNG}

Die in Unternehmen quer durchdringenden Informations- und Kommunikationstechnologien, die spezifisch geschaffen sind für produktive und segmentierte Prozesse, erbringen vor allem in kleineren Unternehmen komplexere Leistungen. Um die Schwierigkeiten beim Management und ihrem Umfeld einzuschätzen haben wir bei 20 Unternehmen die Abläufe der Entscheidungsfindung bei der Umsetzung dieser Technologien analysiert. Die Initialrolle des Geschäftführers und seines externen Umfelds sowie die zustimmende Grundhaltung des ganzen Teams konnte klargestellt werden. Eine Klassifikation der Unternehmen wird vorgeschlagen, die der relativen Bedeutung der verschiedenen Einflussfaktoren Rechnung trägt.

\section{Introduction}

Au cours des deux dernières décennies, les investissements en TIC ont marqué la vie des entreprises, toutes tailles confondues (Sessi, 1999). Les mesures effectuées par l'INSEE, en France, quant à la diffusion des TIC (INSEE, 2000; Crépon et Heckel, 2000) font apparaître l'importance du capital désormais immobilisé (20\% du total des investissements en équipement). Si tous les secteurs d'activité sont concernés (Gadille et d'Iribarne, 2000), les modalités d'usage de ces technologies diffèrent selon les entreprises, et le degré d'appropriation varie considérablement selon les acteurs économiques (Venkatraman et Henderson, 1999).

La généralisation de ces investissements n'a pas eu à court et moyen terme les effets escomptés sur la performance financière et organisationnelle des entreprises. Cette situation a suscité une interrogation, rendue célèbre, sous le nom de paradoxe de Solow ${ }^{1}$; aujourd'hui, quinze ans plus tard, la question du retour sur

1. Économiste honoré par le prix Nobel d'économie, en 1987, qui s'interrogeait sur la faiblesse du retour sur investissement des sommes consacrées à l'équipement informatique. 
investissement des dépenses consacrées à l'informatisation ne trouve encore que des réponses partielles (Askenazy et Gianella, 2000; Croteau, Bergeron et Raymond, 2001 ; Brynjolfsson, 1993 ; Brynjolfsson et Hitt, 1996 ; Monnoyer, 1997). Plusieurs analyses économétriques récentes menées tant en Amérique du Nord qu'en France (Askenazy et Gianella, 2000) font pourtant apparaître le rôle des efforts de réorganisation interne postérieurs aux décisions d'investissements informatiques sur la productivité globale des facteurs. Ces auteurs ajoutent que «l'informatisation seule n'améliorerait en rien les performances des entreprises ».

Lorsqu'on limite le champ d'analyse aux PME, l'impact du recours aux TIC semble encore plus complexe à analyser. Les chiffres révèlent un décalage global entre grandes entreprises et PME (SESSI, 1999) et une forte diversité des situations d'une entreprise à l'autre (Gadille et d'Iribarne, 2000 ; Bergeron et Raymond 1996). Les explications apportées sont nombreuses :

- Une rentabilité des investissements en TIC plus faible dans les PME que dans les grandes entreprises. La différence serait liée à une intégration limitée des PME dans des structures résiliaires (Bergeron et Raymond, 1996).

- Une démarche stratégique qualifiée d'émergente (Julien et Marchesnay, 1988). L'absence de décisions structurées dans une PME freine la mise en place d'une veille qui dépasse le cadre des technologies en usage dans son secteur d'activité. Or on sait que la perception des opportunités doit être préalable à l'adoption d'une innovation (Rogers, 1983).

- La faiblesse de la congruence des investissements en TIC avec la stratégie des PME (Bergeron et al., 1998). L'alignement entre les comportements stratégiques et le choix et la gestion des SI semble ainsi contribuer significativement à la performance organisationnelle (Croteau et al., 2001).

La revue de la littérature nous porte à croire que l'absence d'harmonie entre la réflexion stratégique et la décision d'investir dans ces technologies affecte considérablement tant les résultats que la poursuite de l'exploitation de ces technologies. Nous avons donc orienté notre champ de réflexion sur la démarche décisionnelle des PME en formulant l'hypothèse d'une influence des modalités de cette décision et de sa mise en œuvre sur l'exploitation des potentialités des TI.

Les travaux portant sur le processus de décision dans les PME (Marchesnay, 1992) ou sur la stratégie de ces entreprises (Julien et Marchesnay, 1988) ont montré combien leurs dirigeants étaient soumis à leur contexte socio-organisationnel et mis en évidence les incohérences qui pouvaient en résulter.

L'investissement en TIC présente une originalité par rapport aux autres investissements et en accroît la complexité pour le dirigeant de PME. Ces technologies ouvrent en effet de nouvelles perspectives productives (automatisation de certaines

Revue internationale P.M.E., vol. 15, nos 3-4, 2002 
tâches), des possibilités d'innovation (introduction de prestations informationnelles au cœur ou en périphérie du produit proposé sur le marché) et enfin de nouvelles perspectives organisationnelles (commerce électronique, plates-formes d'achat, etc. ; Croteau et al., 2001). Pour insister sur cette palette d'opportunités, Barras (1986) les qualifie de «permissives ». Le caractère transversal de ces technologies rend leur introduction plus complexe et plus lourde que toutes celles qui ont un usage ciblé, productif par exemple, au sein d'une entreprise donnée. Cette décision d'investissement doit donc être prise dans un contexte général que nous pouvons qualifier encore aujourd'hui de sous-information ou de sous-connaissance.

Ces technologies créent donc des perturbations fonctionnelles et de l'incertitude à tous les niveaux de la hiérarchie. Les changements organisationnels apparaissent plus difficiles à surmonter pour les équipes constituées que les modifications stricto sensu des processus productifs (Akrich, Callon et Latour, 1988).

À travers l'analyse du cheminement de la prise de décision d'investissement en TIC, nous avons donc cherché à savoir si, dans le contexte de cette prise de décision, il n'y avait pas un élément explicatif de la dispersion de l'usage de ces technologies et des résultats obtenus selon les entreprises. Une réponse positive à cette interrogation justifierait un approfondissement des analyses organisationnelles des PME en phase d'investissement en TIC.

Nous avons choisi une méthodologie d'analyse centrée sur l'observation fine du terrain en effectuant des entretiens minutieux avec des responsables rencontrés in situ (annexe I). Notre objectif sera essentiellement de décrypter les conditions de l'émergence de la prise de décision d'investissement et de sa mise en œuvre. Nous analyserons donc d'abord les modalités de la sensibilisation aux TIC dans les entreprises interrogées, puis les formes du cheminement nécessaires à leur implantation et, enfin, les premiers usages qui révèlent la manière dont se déroule l'appropriation et se diffusent les changements.

\section{Choisir les TIC : un investissement hors champ de compétence}

L'originalité d'une décision d'investissement en TIC est liée au fait que le dirigeant, qui est souvent un ingénieur ou un technicien, est amené à prendre une décision ne relevant pas, dans la grande majorité des cas, de son champ de compétence technique direct. À l'exception des entreprises qui se créent autour des TIC, ces équipements constituent généralement des «terra incognita » pour les dirigeants et une partie de leurs équipes. 


\subsection{L'introduction des TIC : une décision qui manque de rationalité}

Sur les 21 PME interrogées, 3 ont été créées pour développer un produit ou un service de type TIC, une autre appartenant au secteur des télécommunications y était sensibilisée, pour les 17 autres et quels que soient les appuis sollicités pour analyser l'intérêt d'une orientation vers ces nouvelles technologies et leurs incidences, le dirigeant s'est senti mal armé face à la décision à prendre. Tous ces dirigeants indiquent avoir pris des contacts, collecté des informations, lu pour enrichir leurs connaissances quant aux usages possibles de l'Internet pour leur entreprise. La moitié de notre population, soit 11 entreprises, disposait en interne d'une personne ayant été recrutée pour ses compétences en informatique ; celle-ci a toujours été largement sollicitée pour répondre aux questions du dirigeant et a présenté des avant-projets.

Il y a donc eu démarche proactive ${ }^{2}$ du dirigeant. Cette démarche peut toutefois trouver sa source dans une initiative personnelle du dirigeant ou être le résultat d'une influence de l'environnement externe ou de l'encadrement. Dans cette phase d'investigation, ce sont les fournisseurs, les clients ou donneurs d'ordres et, enfin, les amis qui sont plus souvent cités comme véhiculant des informations et des avis. Seuls quelques cadres disposant d'une relation plus personnelle avec le dirigeant sont nommés par ce dernier. Analysant les motifs de connexion des PME, Gadille et d'Iribarne, (2000) soulignaient également le caractère très exogène des influences qui s'exercent sur le décideur et la fréquence du motif «pour voir» dans la première connexion à l'Internet. L'influence de l'environnement médiatique (presse quotidienne, télévisuelle ou journaux techniques) ou de l'espace industriel de ces firmes (clients, fournisseurs, réseau de dirigeants locaux) ne doit donc pas être sous-estimée dans la naissance de la curiosité relative aux TIC ainsi que dans la construction d'une réflexion sur les implications organisationnelles et stratégiques de leur usage. La prise de conscience par le dirigeant de la complexité de son environnement, la nécessité d'une écoute intégrant la subjectivité de ses interlocuteurs ou l'intégration de la démarche de réflexion dans son action étaient également relevées par Bergadaa et Vidaillet (1996) ou Laroche (1995). Toutefois, la faiblesse numérique de l'encadrement des PME étudiées accentue l'impact de l'observation de Bergadaa : «le dirigeant se caractérise par sa capacité à créer des processus de décision auxquels il intègre les autres ».

2. La proactivité signifie la volonté de faire, l'existence d'un engagement personnel de l'acteur économique.

Revue internationale P.M.E., vol. 15, nos 3-4, 2002 
Le rôle moteur du dirigeant et les influences externes qu'il reçoit nous semblent donc déterminants dans la prise de décision. Toutefois, l'intensité de sa démarche proactive module l'intensité de la réflexion sur la place des TIC dans l'organisation productive et commerciale.

Nous distinguerons donc quatre degrés d'implication des dirigeants, selon l'intensité modeste ou élevée de la proactivité déployée et le comportement moteur ou suiveur du dirigeant.

Pour faciliter le suivi de notre raisonnement, nous faisons figurer les entreprises interrogées (désignées par leur surnom) et nous qualifions les quatre situations relevées.

TABLEAU 1

Typologie des situations des PME relativement aux TIC selon la proactivité et le comportement du dirigeant

\begin{tabular}{|c|c|c|}
\hline $\begin{array}{l}\text { Comportement } \\
\text { du dirigeant }\end{array}$ & Modeste & Élevée \\
\hline $\begin{array}{l}\text { Dirigeant } \\
\text { initiateur }\end{array}$ & $\begin{array}{l}\text { Akugui, Médoux, Chouèt } \\
\text { et Marquogam. } \\
\mathbf{1} \text { - les curieux }\end{array}$ & $\begin{array}{c}\text { E-c@ve, Gépéto, Montrinfot, Vétimin, } \\
\text { Kadal et Copévin. } \\
\mathbf{4} \text { - les stratèges }\end{array}$ \\
\hline $\begin{array}{l}\text { Dirigeant } \\
\text { suiveur }\end{array}$ & $\begin{array}{c}\text { Renaldia, Infotek, Chocamande, } \\
\text { Batirenov, Arvin et Terrinos. } \\
\text { 2 - les suiveurs }\end{array}$ & $\begin{array}{c}\text { Barbarie, Coupianum, Cendrillon, } \\
\text { Embalto et Laserjet. } \\
\text { 3- les entreprenants }\end{array}$ \\
\hline
\end{tabular}

\section{Situation 1 : les curieux}

L'intuition du dirigeant le conduit à s'intéresser personnellement aux TIC et, en particulier, à l'Internet. Les avantages communicationnels l'interpellent; il prend un abonnement, ouvre une boîte aux lettres, généralement à son nom personnel, et met en place, souvent lui-même ou avec l'aide d'un proche, quelques outils (sites, courrier électronique, ordinateurs en réseau, etc.) sans toutefois modifier l'organisation de son entreprise. Les «curieux » évoquent la difficulté de leur parcours lorsque, s'appuyant principalement sur leur intuition, ils ont dû chercher des fournisseurs, des interlocuteurs aptes à décoder leur perception des usages possibles. Ils justifient ainsi la limitation de leur engagement personnel dans la démarche d'implantation des TIC.

\section{Situation 2 : les suiveurs}

Un donneur d'ordres, un partenaire, un entrepreneur ami fait découvrir au dirigeant le potentiel de l'Internet et les usages qu'il pourrait en tirer pour leur partenariat 
et/ ou le développement de ses relations commerciales nationales ou internationales. Intéressé, le dirigeant donne son accord à la mise en place de certaines applications, parfois très peu nombreuses, en se reposant largement sur les initiatives internes que la décision initiale a suscitées.

Nos interlocuteurs qualifiés de suiveurs n'hésitent pas à citer ceux et celles qu'ils considèrent comme leur initiateur, leur appui. Ils insistent sur le rôle joué dans leur apprentissage par leur réseau professionnel ou les relations amicales qui existent entre dirigeants d'une même ville. Mais la modestie de leur engagement est durement ressentie, voire jugée par leur entourage; elle a parfois meurtri, ou fait partir de l'entreprise, les cadres qui leur avaient emboîté le pas.

\section{Situation 3: les entreprenants}

Le dirigeant intéressé par les avantages communicationnels de l'Internet prend un abonnement et développe, avec l'appui de certains membres de l'encadrement ou de partenaires extérieurs, des applications diversifiées qui font évoluer les pratiques de communication, la circulation interne, voire externe, de certains dossiers. Des projets sont en cours lors de nos visites.

Les dirigeants « entreprenants » évoquent la lourdeur de certaines expériences et les difficultés à concilier la direction de leur affaire et le lancement de certaines initiatives. Ils sont très reconnaissants de l'appui que leur ont apporté, dans ces moments difficiles, leurs salariés les plus dynamiques, quel que soit leur niveau de qualification.

\section{Situation 4 : les stratèges}

Le ou les dirigeants, grâce à leur formation ou à leur ouverture d'esprit, s'intéressent aux TIC et cherchent à les utiliser pour redéfinir leur positionnement stratégique, leurs produits et leur organisation. Les applications, dont ils effectuent ou suggèrent la mise en place, sont multiples; leurs résultats peuvent toutefois être visibles ou seulement attendus.

Ces stratèges évoquent avec un réel enthousiasme les différentes étapes de leur démarche, leurs perspectives à moyen et long terme, même si leurs résultats actuels ne sont pas toujours à la hauteur des investissements personnels et financiers réalisés.

Cette première démarche typologique a été construite sans tenir compte de «l'histoire» de l'implantation des TI dans les entreprises visitées. Or la diffusion de la «seconde vague» des TIC, trente ans après que l'informatique a fait son apparition dans les entreprises, ne peut plus être comparée à celle de l'informatisation des années 1970. Elle s'opère de façon plus équilibrée, semble-t-il, que la première, et ce quels que soient les pays ou les régions. Les dirigeants de PME et

Revue internationale P.M.E., vol. 15, nos 3-4, 2002 
leurs salariés, s'ils n'ont pas toujours une connaissance explicite des TIC, ont souvent une connaissance tacite de certains de leurs usages. Nous avons donc rapproché notre analyse de la typologie de la diffusion des TIC dans le temps et dans l'espace, proposée par Genthon et Arcangeli (1997).

\subsubsection{La prise de décision et les effets de la diffusion des TIC}

Genthon et Arcangeli qualifient la diffusion des TIC de :

- hiérarchique, pour la messagerie interne. Ce qualitatif signifie que la diffusion de la messagerie a eu lieu progressivement, des plus grandes aux plus petites entreprises ;

- déterminée par la masse critique, pour l'Internet. Le raccordement au réseau ne se développe réellement qu'à partir du moment où un très grand nombre d'abonnés rend généralisable l'usage de la toile pour communiquer ou recueillir des informations ;

- à externalité incomplète, pour l'EDI. Les entreprises qui sont intégrées dans des structures résiliaires prennent conscience de l'intérêt de cette technologie, qui reste sans véritable intérêt pour les autres entreprises ;

- dominée par des rétroactions pour les téléservices. La phase d'apprentissage des usagers est déterminante pour la définition des usages finaux par chaque utilisateur.

Sur le champ réduit qui est le nôtre, il nous semble possible de confirmer certains des éléments de cette typologie :

- Toutes les entreprises interrogées ont une adresse électronique, quelle que soit la qualité de leur équipement général en TIC. Cet «investissement » leur est apparu indispensable pour communiquer avec certains clients ou fournisseurs. On peut donc évoquer l'effet de masse critique pour l'Internet ;

- En revanche, seules deux firmes disposent d'une messagerie interne ou d'un intranet. Sans avoir d'importants effectifs salariés, elles emploient en valeur relative un nombre plus grand de cadres et de personnels très qualifiés. On peut donc parler de diffusion hiérarchique pour la messagerie interne. Toutefois, même dans les toutes petites firmes, la pratique de la numérisation de l'information peut conduire à développer un réseau interne (Barbarie). Il en est ainsi lorsque le dirigeant tient à mettre l'information largement à disposition de ses salariés, si un problème, comme par exemple celui de la distance géographique entre l'atelier et le siège, se pose. L'effet de taille minore donc l'usage de la messagerie interne, sauf en cas de proactivité forte de la part du dirigeant;

Revue internationale P.M.E., vol. 15, n ${ }^{\text {s }} 3-4,2002$ 
- Nous constatons aussi l'existence d'une diffusion à externalité incomplète et l'influence du réseau de relations. Ainsi, la seule entreprise qui fonctionne sous EDI (Terrinos) est liée pour la commercialisation de ses produits à la grande distribution qui l'a effectivement poussée vers ce mode de passation des commandes. A contrario, l'absence d'exigences des donneurs d'ordres ou des partenaires principaux réduit la proactivité des dirigeants (Infotek, Chocamande), alors que l'affirmation des besoins dynamise cette proactivité (Laserjet);

- Nous vérifions enfin le rôle d'apprentissages antérieurs et l'existence d'une diffusion dominée par des rétroactions. Toutes les entreprises qui avaient utilisé le minitel, pour échanger des données avec des administrations par exemple, sont passées «naturellement» aux fichiers téléchargés (Batirenov) et aux documents attachés dans le courrier électronique (Laserjet). A contrario, celles qui n'ont pas dépassé le fax comme mode de transmission des informations interentreprises n'ont pas recours aux téléservices, ni au téléchargement de documents administratifs (Infotek, Chocamande).

Genthon et Arcangeli (1997) constatent une influence des secteurs d'activité sur la diffusion des TIC. Nos observations sont plus nuancées sur ce point. Ainsi, nous avons relevé que les modes de communication intrasectorielle influent sur la diffusion des TIC, mais que le comportement des donneurs d'ordres importants peut faire obstacle aux modes de fonctionnements sectoriels (Infotek). De même, la dynamique de certains dirigeants peut contraster avec les habitudes du secteur (Chouet, Batirenov, Vetimin).

Les réflexions de Genthon et Arcangeli nous permettent de préciser ce que, de façon très générale, nous avions appelé «influence de l'environnement». Nos propres observations nous conduisent à rappeler que, dans les PME, la personnalité du dirigeant, et en particulier sa proactivité, peut lui permettre de dépasser les incidences sectorielles, voire les contraintes liées à la taille.

Ces différents éléments nous incitent à affiner notre typologie en quatre groupes.

- Nous distinguerons ainsi, de l'ensemble des suiveurs, le cas de Terrinos, très marqué par son appartenance à un réseau de distribution ;

- Nous rapprocherons quatre entreprises - Chouet, Vetimin, Barbarie et Cendrillon - dans lesquelles la dynamique et la réflexion du dirigeant quant au potentiel des TIC ne se relâchent pas au fil du temps ; notons qu'elles ne se matérialisent pas avec autant d'efficacité dans chaque cas. La très petite taille de l'entreprise (effet taille) limite les moyens matériels et freine les ambitions (Chouet et Barbarie). La stagnation du marché (effet sectoriel) contraint à ralentir la mise en œuvre des projets (Vetimin et Cendrillon).

Revue internationale P.M.E., vol. 15, nos 3-4, 2002 
Pour en rendre compte, nous regroupons, dans la figure 1 ci-après, les entreprises dont les comportements sont les plus voisins.

FigURe 1

\section{Comportement des entreprises}

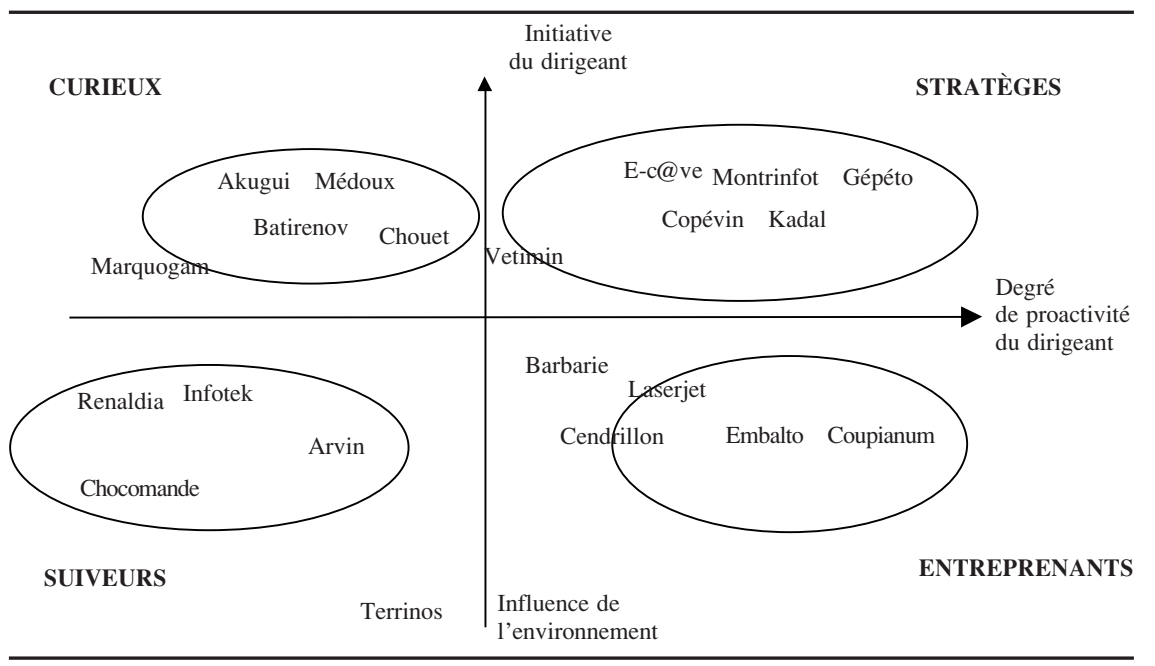

\section{Le cheminement technologique}

Les conclusions des travaux portant sur le lien entre caractères de l'entreprise et degré d'informatisation convergent sur la moindre accession des PME aux technologies de production avancées (Grenam, 1996, pour la France; Julien, 1995, pour le Canada) et sur leurs usages en termes d'organisation (Bergeron et Raymond, 1996, au Canada; SESSI, ministère de l'Industrie, 2000, pour la France).

Pour expliquer ce «retard»global et les écarts importants que l'on peut relever dans l'ensemble des PME, on invoque souvent l'impact de la faiblesse des effectifs sur le degré de qualification des personnels. On trouverait ainsi des entreprises qui, dotées d'équipements très performants et d'un personnel de forte qualification, pourraient choisir des stratégies de positionnement technologique très avancé. Parallèlement existeraient des entreprises pénalisées dans la mise à jour technologique et l'exploitation stratégique par la faiblesse de leurs fonds propres et le petit nombre de leur personnel hautement qualifié. Ces personnels n'auraient pas une compétence en informatique leur permettant d'en projeter les usages productifs et organisationnels (Girard, 1997; Grenam, 1996).

Nous avons mis en évidence, dans la phase de sensibilisation, l'influence du dirigeant, mais aussi celle de son environnement extérieur, ainsi que le rôle de 
caractéristiques structurelles telles que la taille. À ces éléments pourraient donc s'ajouter, dans la phase d'appropriation des TIC : 1 ) des effets liés à la qualification du dirigeant et de son équipe dont font état les travaux cités ; 2) des effets liés à la taille de l'entreprise.

Nous avons donc observé les savoir-faire disponibles dans les entreprises observées (formation initiale et compétences acquises sur le terrain) et affiné notre analyse de l'effet taille pour vérifier la prégnance de la dichotomie annoncée.

\subsection{La qualification des équipes : une condition nécessaire et / ou suffisante?}

Deux exemples contrastés sur le champ observé ont retenu notre attention.

1. Renaldia dispose d'un informaticien et de plusieurs cadres rompus à l'usage des TIC; ils ont incité le dirigeant à équiper l'entreprise d'un outil informatique structuré en réseau. Toutefois, même après plusieurs années d'utilisation, le dirigeant ne perçoit pas le potentiel des outils informatiques et en conteste l'efficacité. Il exige que toutes les données et résultats soient imprimés et opère un contrôle manuel sur les listings. Enfin, il véhicule un discours si négatif sur les usages que l'informaticien ne veut plus proposer de nouvelles implémentations sans les avoir soumises à un cabinet de consultants, susceptible de valider son analyse. A contrario, les dirigeants de Vetimin, à la suite d'une émission de télévision grand public sur les fonctionnalités de l'Internet, ont saisi les avantages qu'ils pourraient tirer de la création d'un site Web. Ils ont développé en un temps record une commercialisation aux particuliers, distincte des marchés publics qui constituaient l'essentiel de leur clientèle.

La qualification des équipes ne semble pas constituer une condition suffisante pour la mise en œuvre d'applications des TIC. L'intuition du dirigeant et sa sensibilité aux évolutions nous semblent influencer fortement sa démarche stratégique, quels que soient les moyens humains dont il dispose. Le contraste entre les deux situations présentées avait déjà été relevé par d'autres auteurs.

Ainsi, analysant l'intégration des TIC dans la PME, Harrison, Mykytyn et Riemenschneider (1997) constataient que l'influence exercée par les dirigeants, ou les possesseurs du capital, dépendait essentiellement de la perception (et non de la connaissance) qu'ils ont de l'usage des TIC et de la prégnance des difficultés financières perçues et appréhendées.

Si une perception positive permet de surmonter les contraintes financières, une situation financière réduit considérablement l'intérêt porté aux technologies. Julien et al. (1995) vont dans le même sens et soutiennent que la veille technologique

Revue internationale P.M.E., vol. 15, nos 3-4, 2002 
constitue un effet de la définition d'une stratégie, et non l'inverse. C'est le caractère proactif et stratège du dirigeant qui le pousse à la veille informationnelle et à l'implantation de nouvelles solutions, plus que son niveau de formation ou sa spécialisation initiale.

2. Chez Akugui, nous avons observé que c'est la décision de développer des contacts à l'extérieur de la France qui a incité le dirigeant à analyser les modalités de fonctionnement du Web, puis à engager une personne (formation de technicien en alternance en commerce international) pour mettre à jour le site et traduire les pages en plusieurs langues, etc. De même, chez Arvin, la décision d'élargir l'activité au marché international, du fait de la concurrence nationale, a conduit le dirigeant à s'intéresser à l'Internet, au logiciel Lotus Notes, etc. En revanche, chez Infotek (90 employés, dont un nombre important d'ingénieurs et de techniciens très qualifiés), la position de sous-traitant d'un acteur majeur du secteur des télécommunications et les nombreux contacts entre les deux firmes sur le plan local ne se traduisent pas par la mise en place d'une veille. Les responsables se sentent d'ailleurs incapables de rédiger un cahier des charges en matière d'équipement Internet parce qu'ils n'arrivent pas à déterminer l'utilisation qu'ils en feraient.

Dans l'ensemble des PME, la proactivité du dirigeant semble compenser la faiblesse de sa formation technologique et même celle de ses salariés. En revanche, ses hésitations ne permettent pas aux salariés compétents de donner toute leur mesure.

Au sein d'une PME, les échanges informels, la diffusion des connaissances tacites semblent plus aisés et naturels que dans des entreprises de taille importante supposant une structuration plus affirmée. Les travaux sur la diffusion de l'innovation dans les entreprises montrent toutefois que cette affirmation doit être nuancée.

\subsection{Le rôle des intermédiaires, ces indispensables porte-parole...}

L'appropriation des technologies «not invented here ${ }^{3}$ » (Durand, 1988), la diffusion de nouvelles méthodes de travail (Venkatraman, 1991) et la mise en œuvre d'une stratégie nouvelle (Alter, 1995) se heurtent toujours à des réticences, quelles que soient la taille de l'entreprise ou la nature de la technologie. Les réflexions sur la diffusion interne de l'innovation mettent en avant le rôle des «porte-parole» (Akrich, Callon et Latour,1988) qui transcrivent des perceptions générales en questions plus concrètes, des porteurs de projet qui diffusent dans l'entreprise les caractéristiques d'une innovation et apaisent les craintes qu'elle fait naître... En l'absence de ces intermédiaires qualifiés ou sécurisants, le projet ne se développe pas.

3. Anglicisme qui indique l'origine externe de la technologie.

Revue internationale P.M.E., vol. 15, ns 3-4, 2002 
L'existence de ces porteurs de projet peut-elle constituer, dans les PME qui ne disposent pas d'effectifs très qualifiés, un catalyseur de la proactivité du dirigeant?

- Chez Copévin qui ne compte que 22 salariés permanents, et aucun informaticien, «il y a bien longtemps qu'on n'a pas vu les prestataires, on est sur Internet et par télétransmission ». La saisie de la commande enclenche les tâches logistiques, la facturation, la comptabilité, etc. Mais, l'informatique est présente dans l'entreprise depuis 1982, le «SI est perpétuellement inachevé et nous, en formation». De même chez Vetimin, les projets des dirigeants ont été dynamisés par la réaction de certains membres du personnel, de qualification ouvrière, qui ont cherché à transformer les processus existants pour les adapter à une production individualisée à la commande.

- En revanche, chez Terrinos (42 employés), c'est le distributeur qui a imposé l'utilisation de l'EDI, il y a maintenant cinq ans. Si l'entreprise a réalisé un investissement important pour se doter d'un logiciel de gestion commerciale, les bons de commande EDI sont toujours ressaisis et envoyés sous format PDF toutes les demi-heures, etc. Mais, « on a des problèmes de relation avec la société d'informatique, c'est un copain du PDG ». Les applications n'ont donc pas suivi, faute d'appui interne, et sans doute à cause du mauvais choix de partenaire.

L'existence, au sein de la structure, de relais qui s'investissent dans le projet et agissent, nous semble-t-il, comme des renforts de la proactivité du dirigeant et de sa capacité à s'approprier une technologie nous incite donc à revoir l'appellation de l'axe horizontal de notre schéma, soit le degré de proactivité de l'entreprise (et non plus du seul dirigeant).

Ce rôle de l'entourage interne du dirigeant nous semble rendre moins prégnante la question du niveau de formation, qu'il s'agisse de celle du dirigeant (Bergeron et Raymond, 1996) ou des salariés (Grenam, 1996). Toutefois, il nous paraîtrait téméraire de négliger les effets de seuil que peut poser la taille de l'entreprise sur la présence de ces porte-parole, de ces porteurs de projets. La situation évoquée précédemment, par l'entreprise Chouet par exemple, nous rappelle la difficulté des dirigeants des toutes petites entreprises à s'abstraire des contraintes de la production pour mettre en œuvre les stratégies innovantes qu'ils ont conçues.

\subsection{Peut-on être petit?}

Les recherches portant sur les PME se butent souvent à la définition de seuils exprimés en nombre de salariés. Des chiffres tels que 200 salariés ou 50 salariés sont souvent cités pour expliquer le besoin de structures différentes par rapport à

Revue internationale P.M.E., vol. 15, nos 3-4, 2002 
un effectif considéré comme significatif du terme de PME de moins de 500 salariés (Mérigot, 1991 ; Julien, 1994 ; Le Vigoureux, 1995 ; Kalika, 1991). Notre champ d'observation se situe dans les tranches basses (moins de 100 salariés). Les réflexions de Boutary (2001), qui a travaillé sur le même échantillon d'entreprises, font apparaître le caractère complexe de la notion de proximité et des liens qui unissent le dirigeant aux membres de son réseau interne et externe. Il semble toutefois que l'usage des TIC favorise le recours fréquent à ces réseaux, voire celui des salariés à leur propre réseau, et allège les incidences de la faiblesse des formations.

Mais les contraintes que fait peser la petite taille des établissements ne se manifestent pas que sur le niveau de formation. Comme nous le notions avec Genthon et Arcangeli ou Harrison précédemment, la faiblesse des effectifs semble freiner, malgré la proactivité du dirigeant, la diffusion des TIC au sein de la PME. Plusieurs explications à cette situation sont apportées par la littérature :

- Premièrement, Bergeron et Raymond (1996) constatent que les incidences positives de l'utilisation de l'EDI sont plus faibles dans les PME que dans les grandes entreprises. Ces auteurs montrent que la faiblesse du nombre des fonctions internes utilisant l'EDI y est moindre; l'influence des donneurs d'ordres y explique plus souvent le recours à l'EDI que dans les grandes structures. Malgré cela, les réductions de coûts y sont sensibles.

- Deuxièmement, l'attraction qu'excercent les TIC sur les PME ne serait pas de la même ampleur que sur les firmes de taille plus importante, les principaux effets des TIC portant sur la gouvernance des entreprises et les relations intrafirmes (Galliano, Roux et Filippi, 2000). En outre, une complexité fonctionnelle accrue et l'existence de processus collectifs d'organisation du travail augmentent l'intérêt du recours aux TIC, puisqu'elles permettent de réduire les coûts de transaction et de coordination interne.

- Troisièmement, la faiblesse de la réflexion stratégique et de l'incorporation des TIC dans la structure productive et commerciale, liée à la position de suiveur de certains dirigeants de PME, rend moins manifestes les incidences de l'usage de ces technologies. Par là même, elle les fait hésiter à reconsidérer leurs choix stratégiques, ou à dynamiser leurs équipes.

Ces conclusions, qui s'appuient sur des analyses statistiques solides, ne sauraient être remises en cause, mais les PME d'aujourd'hui sont de plus en plus souvent confrontées avec des problèmes de gouvernance et des structures intrafirmes. Ainsi, nous avons assisté en France, au cours de la décennie 1990, à une véritable explosion du nombre des microgroupes. Entre 1990 et 1998, le nombre des PME dépendant d'un tel groupe a été multiplié par cinq : 9822 en 1998 contre 2180 en $1990^{4}$. La structure des microgroupes peut être complexe, alliant plusieurs

4. Notes bleues de Bercy no 206, $1^{\text {er }}$ mai 2001.

Revue internationale P.M.E., vol. 15, nºs 3-4, 2002 
niveaux de contrôle et une implantation dans plusieurs domaines d'activité; leur gestion et leur organisation font dès lors apparaître des besoins similaires à ceux des structures de grande taille.

Les observations menées sur notre échantillon d'entreprises confirment cette évolution.

- Le développement du travail en partenariat avec des fournisseurs chez Terrinos, ou au sein du groupe de PME auquel appartient désormais Kadal, a suscité une réflexion sur les implications organisationnelles du partenariat, conduit à en mesurer les coûts et dynamisé l'informatisation des deux entreprises. Chez Kadal, un informaticien a été recruté pour l'ensemble du groupe, constitué de quatre entreprises peu distantes géographiquement. Cette personne a uniformisé les matériels et les logiciels pour faciliter la mise en place de passerelles entre les fichiers des différents services et entre les différentes PME du groupe. Son objectif est désormais d'introduire un progiciel de gestion intégrée au sein du groupe.

- Chez Arvin, l'appartenance à un groupe constitué de plusieurs établissements éloignés les uns des autres a incité l'entreprise à se doter du logiciel Lotus Notes, pour faciliter la transmission des éléments financiers et la définition d'un agenda commun. Mais si les services comptables en sont de grands utilisateurs, l'intérêt de la fonction « agenda » ne fait pas l'unanimité dans l'ensemble de l'établissement ; cette fonction n'est guère utilisée, le personnel destinataire n'ayant que fort peu de réunions communes.

- On retrouve chez Chocamande une situation similaire. La société, qui connaît une très forte croissance justifiant une augmentation importante des moyens de production, n'a pas encore "pris le temps » d'organiser autrement que par le fax et le téléphone les contacts avec l'autre unité de production du groupe. Ces relations s'appuient essentiellement sur des contacts personnels entre le président du groupe et le dirigeant de l'établissement que nous avons visité. Entre eux, le fax relaie l'oralité distante, lorsque cela se révèle nécessaire.

Plus que la taille de l'entreprise, c'est son mode de fonctionnement et son organisation qui font naître les besoins en TIC; les dirigeants proactifs ou les membres de l'entreprise curieux y font référence dès que les relations qu'entretient la PME avec ses partenaires ou ses alliés se densifient et se structurent.

Une fois la première décision prise, le cheminement vers des applications et des usages de plus en plus variés suppose que s'exercent auprès du dirigeant:

- des influences extérieures qui viendront le conforter et l'aider;

- l'action des cadres et salariés de l'entreprise qui développeront applications et procédures quotidiennes ou structurantes.

Revue internationale P.M.E., vol. 15, nos 3-4, 2002 
La figure 2, présentée ci-après, met en valeur le jeu de ces différentes influences sur les terrains étudiés et nous permet d'affiner notre typologie.

FIGURE 2

Le cheminement des décisions et usages des TIC dans les PME

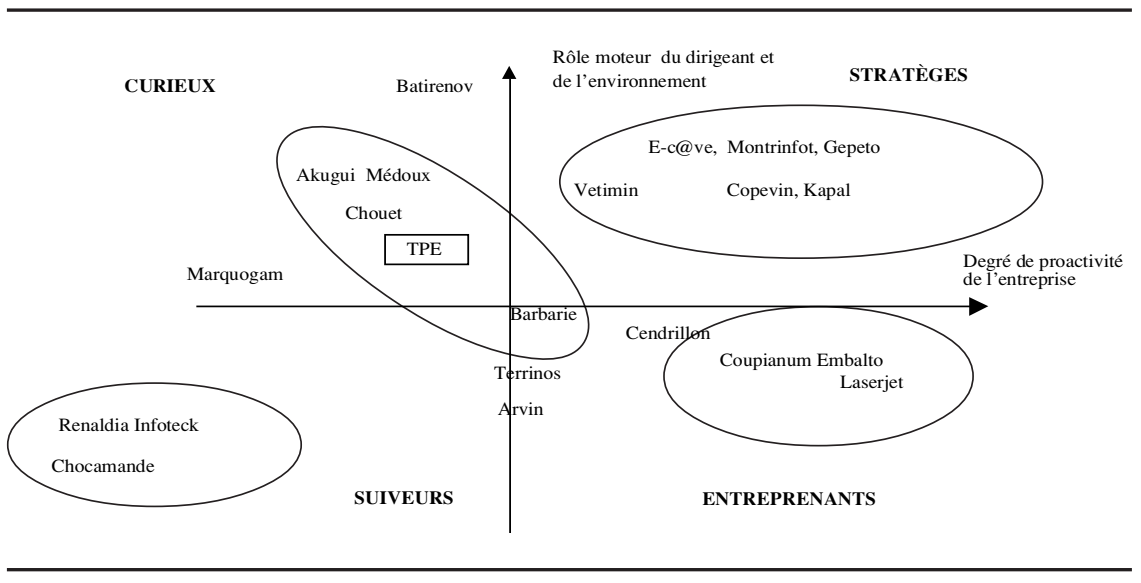

\section{De la décision d'investir à la multiplication des usages}

Contrairement à ce qui s'est produit lors de la diffusion des outils informatiques, rares sont les réticences marquées à l'égard de l'utilisation de l'Internet, des cédéroms ou autre webcam. L'interconnexion entre les usages privés et professionnels, et l'appropriation par de nombreux jeunes en dédramatisent l'usage et en élargissent les occasions d'apprentissage.

Arthur et al. (1987) formalise cette dynamique des processus de diffusion technologique à l'aide de la notion de rendements croissants et, en particulier, de rendements croissants d'information. Plus une technologie est adoptée, moins l'aversion au risque constitue un facteur de blocage à sa diffusion (Foray, 1989). Notre réflexion nous a permis de moduler les conclusions apportées par certains auteurs aux effets contraignants de la taille et de la faiblesse des formations sur la diffusion dans les PME de TIC. Toutefois, la faiblesse de la taille peut favoriser ou au contraire réduire l'impact de l'aversion au risque de certains personnes influentes dans l'entreprise (Médoux, Renaldia...) et dès lors agir sur le rythme de diffusion d'une nouvelle technologie et de ses applications. Nous avons donc cherché sur nos terrains d'observation à qualifier les modalités de cette diffusion, à préciser les éléments favorables au développement des usages des TIC et les freins qui demeurent. 


\subsection{Les modalités de la diffusion interne: bricolage, dilution ou stratégie?}

Dans toutes les PME interrogées ${ }^{5}$, les utilisateurs insistent sur la parenté entre minitel et Internet. Les modes de connexion, la présentation des informations sur un site, l'usage de l'écran n'y apparaissent pas plus complexes, au contraire; la télétransmission est aussi plus simple. Le minitel semble bien avoir constitué, dès lors, une étape de formation particulièrement importante pour les personnels administratifs et de secrétariat qui utilisaient cet outil bien plus que les cadres et dirigeants de PME (Monnoyer, 1990).

- Chez Batirenov, l'assistante de l'un des dirigeants qui exerce aussi la responsabilité de comptable s'émerveille du temps gagné par la télétransmission des déclarations annuelles de salaires. Elle n'hésite pas à travailler en double sur les versions nouvelle et ancienne du logiciel de comptabilité pour débusquer les «bugs » de la dernière mouture.

- Chez Coupianum, la commerciale, en poste depuis vingt ans, enrage qu'on ne remette pas plus souvent à jour le site. "Les images, cela impressionne, un tout petit pays de l'ex-URSS nous a trouvés, cela m'épate.» Les expériences réussies constituent aussi des incitatifs pour la mise en place de nouveaux usages, très différents parfois d'un simple envoi ou rapatriement de fichiers. Chez Kadal, le secrétariat commercial admet difficilement que l'intégration des fichiers qui leur est nécessaire pour répondre rapidement et de façon précise aux transporteurs ou aux clients soit toujours retardée en raison des tâches demandées par la comptabilité à l'informaticien. Le dirigeant de Barbarie, qui a développé sa communication clients grâce aux sites et au courrier électronique, affine cette communication en travaillant sur plusieurs sites qui visent à répondre à des demandes de publics différents et envoie par ailleurs une lettre électronique trimestrielle.

- Le comptable de Copévin est devenu, avec le temps et de nombreuses formations, responsable informatique. Il met sans cesse en place de nouvelles applications en réseau. Une situation similaire se retrouve chez Laserjet où le responsable de la production insiste sur le fait que «nous nous sommes un peu formés nous-mêmes ». Chacun puise dans les TIC pour monter, à l'aide d'une webcam, une visite de l'usine en direct et à distance, réaliser un cédérom envoyé par la poste pour présenter la

5. Comme nous l'avons mentionné plus haut, nos terrains ne comptent que deux entreprises dont les processus sont liés intrinsèquement aux TIC. Dans celles-ci, les questions d'adaptation se poseront plus concrètement dans les années à venir.

Revue internationale P.M.E., vol. 15, nos 3-4, 2002 
production, travailler avec les clients qui envoient désormais leurs plans en fichier attaché, ce qui permet d'éviter la reprise des dessins et des cotes. Un foisonnement d'idées et leur mise en œuvre sont proposés au dirigeant par les cadres et validés par ce dernier.

Cette validation par le dirigeant d'applications quelquefois hétérogènes ne constitue pas une stratégie au sens propre du terme, mais une réponse à la difficulté spécifique que représente pour les PME industrielles l'appropriation d'une technologie transversale à leur métier. Le terme de bricolage employé par Ciborra (1993) au temps du minitel semble encore d'actualité. Elle met aussi en évidence le rôle majeur des «porteurs de projet » qui s'approprient le changement technique et l'intègrent dans leur travail. Comme l'usage des TIC introduit des innovations, et repose sur une transformation culturelle, « les directions ne décident pas en la matière, mais régulent, articulent et apprennent» (Alter, 1995). La pratique de tâches diversifiées et de responsabilités multiples semble bien favoriser la réactivité des salariés devant une telle technologie.

\subsection{Peut-on aller plus loin?}

La connexion à l'Internet, générale dans toutes les entreprises visitées, a suscité une curiosité qui a largement facilité le développement de son usage, dès que les inquiétudes, du dirigeant ou d'un cadre de haut niveau, n'en freinaient pas la diffusion. L'aisance n'est cependant pas générale pour l'ensemble du personnel et porte principalement sur un usage courant dont l'amplitude peut être restreinte pour des raisons diverses.

- La limitation du nombre des adresses (octroi d'adresses aux seuls postes d'encadrement ou de communication) est souvent présentée comme une nécessité pour parer aux risques que ferait encourir la connexion sur l'extérieur du réseau informatique interne. Chez Médoux, la dirigeante, très marquée par les observations de son comptable sur les risques d'intrusion de «hackers » dans ses fichiers, a attendu que le comptable parte à la retraite pour mettre en place une liaison Internet et le courrier électronique. Ensuite, elle a limité à un poste l'ouverture vers l'extérieur, les autres PC fonctionnant en réseau.

Au-delà des problèmes réels de sécurité, ce type d'inquiétude reflète des méconnaissances profondes des potentialités des TIC chez les cadres (dirigeants et non dirigeants) qui freinent la dynamique des usages.

- Chez Chocamande, le directeur de l'établissement qui jongle avec aisance sur ses tableaux Excel ne dispose pas sur son propre ordinateur d'une adresse électronique. C'est sa secrétaire, dans le bureau voisin, qui est responsable du courrier qu'il soit papier ou électronique.

Revue internationale P.M.E., vol. 15, nºs 3-4, 2002 
La question du partage de l'information stratégique, du travail collectif sur des informations «sensibles» sur le plan de la gestion, ne semble pas encore être posée.

- Chez Renaldia, entreprise exportatrice ayant des filiales à l'étranger, aucun ordinateur visible dans le bureau du dirigeant qui assume pourtant des fonctions de représentation non négligeable à la Chambre de commerce et d'industrie et dans des organismes régionaux. Seul un vieux téléphone lui permet d'interroger son responsable informatique lorsqu'il vise quotidiennement les listings de sortie de production.

Mais l'appropriation généralisée des nouveaux outils exigerait bien davantage qu'une décision d'investissement.

- Chez Arvin, l'entreprise s'est dotée d'un site, de nombreuses adresses électroniques, d'un intranet et d'un informaticien. Si la responsable export et les services financiers sont de grands utilisateurs, ce n'est pas le cas du responsable des commerciaux France. L'informaticien ne se sent ni soutenu, ni dirigé. «Si je ne mets pas le site à jour, ils ne s’en aperçoivent pas... »

L'appropriation technologique apparaît donc très différente selon les individus au sein d'une même entreprise. Dans un contexte qui se caractérise par une absence de blocage en aval, au niveau des secrétariats et techniciens, le rôle des cadres et responsables de service semble déterminant pour assurer la diffusion organisationnelle des TIC.

Ces observations renforcent le bien-fondé des critères choisis pour notre construction typologique, soit le niveau d'initiative du dirigeant et le degré de proactivité de l'entreprise.

\section{Conclusion}

En montrant que la veille informationnelle était le résultat d'une décision stratégique plus qu'un préalable pour les dirigeants de PME, Julien et al. (1995) laissent percevoir la complexité que représente pour les dirigeants de ces entreprises la décision d'investir dans les TIC. Ces technologies ne sont pas directement liées à leurs savoir-faire professionnels, la perception de leur intérêt et de leur utilité en est donc plus problématique, hors d'une démarche de collecte systématique d'informations et d'expériences.

Dans un contexte encore largement marqué par la question de l'efficience et de la performance des TI (Croteau, Bergeron et Raymond, 2001), nous nous sommes interrogés sur l'incidence des modalités de la prise de décision des dirigeants de PME sur les usages de ces technologies et leur appropriation dans ces entreprises.

Revue internationale P.M.E., vol. 15, $\mathrm{n}^{\text {os }} 3-4,2002$ 
- Le rôle moteur des dirigeants et le degré de leur proactivité sont apparus déterminants sur le terrain aussi bien à l'origine des projets d'investissement en TIC que dans leur développement et leur mise en œuvre. Les inquiétudes des responsables de PME face à la complexité ressentie de la décision à prendre les rapprochent des dirigeants des grandes structures, mais la faible taille de leur entreprise accentue le sentiment d'isolement que suscitent les difficultés d'analyse des problèmes.

- L'environnement relationnel des dirigeants exerce un rôle prépondérant sur la prise de décision. Des pressions externes s'expliquent par les caractéristiques de la structuration du groupe ou du marché auquel appartient l'entreprise (Bergadaa et Vidaillet, 1996). Mais des influences, qui peuvent être décisives, proviennent des hommes et des femmes avec lesquels ces responsables communiquent et constituent des communautés plus marquées par la convivialité et la confiance que dans les structures de grande taille. L'encadrement, soumis aux avancées ou reculades des dirigeants, soutient ou restreint les formes et le développement du projet TIC.

- Le niveau de qualification des salariés apparaît comme un facteur favorable à l'appropriation des technologies comme l'évoquaient les conclusions de Bergeron et Raymond, (1996), mais sa faiblesse peut être dépassée dans des économies dans lesquelles formations et informations sont aisément accessibles, à partir du moment où la direction de la PME et des porte-parole efficaces entraînent les équipes.

- La diffusion des technologies de l'information au sein des PME bénéficie de la curiosité qu'elles suscitent dans le corps social, peu importe la formation initiale et les catégories socioprofessionnelles. Toutefois, cette curiosité n'aura pas d'effets durables si l'encadrement de l'entreprise ne s'implique pas directement dans la mise en œuvre des applications.

Ces observations nous ont amenée à proposer un classement des formes et des modalités d'engagement des entreprises dans l'usage et l'appropriation des TIC. Cette typologie fait ressortir les éléments suivants :

- L'existence de quatre situations différentes qui correspondent aux attitudes des dirigeants que nous avons distinguées comme suit: les suiveurs, les curieux, les entreprenants et les stratèges. La très petite taille et les difficultés conjoncturelles du secteur peuvent toutefois freiner les dynamiques mises en place;

- L'importance de l'initiative du dirigeant dans la décision d'ouverture aux TIC met en relief le besoin d'appui informationnel et matériel des dirigeants pour que leur réflexion prenne une forme plus stratégique qu'intuitive ;

Revue internationale P.M.E., vol. 15, n ${ }^{\text {s }} 3-4,2002$ 
- Le rôle de la proactivité de l'ensemble de l'entreprise sur la dynamique des implantations TIC montre l'importance du besoin spécifique d'écoute de l'encadrement qui exprime les modalités individuelles de l'appropriation technologique. Il souligne aussi l'intérêt que présente la création ou la dynamisation d'espaces de partage de savoirs tacites des communautés qui constituent l'environnement relationnel choisi par les dirigeants des PME.

\section{AnNeXe I}

\section{Méthodologie}

Entre les chercheurs analysant l'impact des TIC sur les choix organisationnels et stratégiques des entreprises, un certain consensus semble se dessiner actuellement quant aux démarches méthodologiques à adopter (Fabbe-Costes, 2000; Bergadaa et Von Burg, 2000). Le recours à une observation fine des usages des acteurs économiques de différents niveaux de responsabilité est préféré à la démarche d'enquête postale, la diversité des fonctions des utilisateurs potentiels rendant difficile la collecte des données par écrit. Les analystes de l'observation insistent par ailleurs sur l'intérêt de partir des innovations pour dresser une liste d'entreprises plutôt que de sélectionner un échantillon d'entreprises afin de repérer celles qui sont innovantes (Le Bas et Torre, 1993). Cette seconde démarche se révèle toutefois moins précise et les résultats statistiques obtenus sur des échantillons importants par Gadille et d'Iribarne (2000) incitent d'ailleurs à un affinement de l'observation. Notre objectif étant de comprendre comment s'opère la prise de décision d'investissement puis d'usage des TIC, l'adoption des principes d'observation choisis par March (1981) nous a semblé adaptée.

Entre décembre 1999 et décembre 2000, 21 entreprises ont fait l'objet de 42 entretiens auprès des dirigeants, des responsables commerciaux et des responsables informatiques, si possible, selon la structure hiérarchique de l'entreprise et la réalité des usages des TIC. Les entretiens menés par deux chercheurs - d'une durée moyenne d'une heure et demie environ - ont été enregistrés, puis entièrement retranscrits pour en permettre l'analyse par les différents membres de l'équipe.

Les entreprises qui ont été choisies répondent aux exigences de représentativité de l'objet de recherche qui sont plus théoriques que statistiques. Nous avons pris en considération aussi bien l'homogénéité que la variété des cas dans la constitution de cet échantillon (Hlady Rispal, 2002).

Les traits communs de notre échantillon sont : une certaine homogénéité des équipements TIC utilisés, la localisation régionale et la taille des entreprises.

La variété de notre échantillon réside dans :

Revue internationale P.M.E., vol. 15, nos 3-4, 2002 
- le niveau d'intégration des outils de télétransmission : certaines entreprises étaient des précurseurs à leur utilisation, d'autres ont une expérience moins importante ;

- la formation des dirigeants : sont également représentées dans notre échantillon les entreprises dont les dirigeants sont dotés d'une solide formation initiale en TIC, d'autres qui l'ont acquise par la pratique et, enfin, d'autres qui n'ont que des connaissances éparses en la matière.

\section{Présentation de l'échantillon}

\begin{tabular}{|c|c|c|}
\hline Entreprises & Secteur d'activité & Personnes interrogées \\
\hline $\begin{array}{l}\text { Cas } n^{\circ} 1 \\
\text { Chouet }\end{array}$ & Confection & Directeur \\
\hline $\begin{array}{l}\text { Cas no } 2 \\
\text { Batirenov }\end{array}$ & Bâtiment & $\begin{array}{l}\text { Directeur et } \\
\text { responsable comptable. }\end{array}$ \\
\hline $\begin{array}{l}\text { Cas } n^{\circ} 3 \\
\text { Vetimin }\end{array}$ & Confection & Directeur \\
\hline $\begin{array}{l}\text { Cas n }{ }^{\circ} 4 \\
\text { Montrinfot }\end{array}$ & Imagerie & Directeur \\
\hline $\begin{array}{l}\text { Cas no } 5 \\
\text { Marquogam }\end{array}$ & Maroquinerie & $\begin{array}{l}\text { Directeur, } \\
\text { responsable informatique et } \\
\text { responsable commercial. }\end{array}$ \\
\hline $\begin{array}{l}\text { Cas } n^{\circ} 6 \\
\text { Gépéto }\end{array}$ & Informatique & $\begin{array}{l}\text { Responsable veille technologique et } \\
\text { secrétaire de direction. }\end{array}$ \\
\hline $\begin{array}{l}\text { Cas } n^{\circ} 7 \\
\text { Chocamande }\end{array}$ & Confiserie & Directeur \\
\hline $\begin{array}{l}\text { Cas } n^{\circ} 8 \\
\text { Embalto }\end{array}$ & Emballage & Directeur \\
\hline $\begin{array}{l}\text { Cas n }{ }^{\circ} 9 \\
\text { Kadal }\end{array}$ & Articles ménagers & $\begin{array}{l}\text { Directeur, } \\
\text { responsable informatique et } \\
\text { assistant commercial. }\end{array}$ \\
\hline $\begin{array}{l}\text { Cas no } 10 \\
\text { Médoux }\end{array}$ & Mégisserie & $\begin{array}{l}\text { Responsable commercial et } \\
\text { responsable télématique. }\end{array}$ \\
\hline $\begin{array}{l}\text { Cas } n^{\circ} 11 \\
\text { Arvin }\end{array}$ & $\begin{array}{l}\text { Fabrication de } \\
\text { capsules }\end{array}$ & $\begin{array}{l}\text { Directeur et } \\
\text { responsable export. }\end{array}$ \\
\hline $\begin{array}{l}\text { Cas n }^{\circ} 12 \\
\text { Laserjet }\end{array}$ & Découpe au laser & $\begin{array}{l}\text { Directeur et } \\
\text { directeur de production. }\end{array}$ \\
\hline $\begin{array}{l}\text { Cas n }{ }^{\circ} 13 \\
\text { Akugui }\end{array}$ & Paramédical & $\begin{array}{l}\text { Directeur et } \\
\text { responsable commercial. }\end{array}$ \\
\hline
\end{tabular}


Présentation de l'échantillon (suite)

\begin{tabular}{|c|c|c|}
\hline Entreprises & Secteur d'activité & Personnes interrogées \\
\hline $\begin{array}{l}\text { Cas no } 14 \\
\text { E-c@ve }\end{array}$ & $\begin{array}{l}\text { Commercialisation } \\
\text { de vins }\end{array}$ & Directeur \\
\hline $\begin{array}{l}\text { Cas no } 15 \\
\text { Terrinos }\end{array}$ & Charcuterie & $\begin{array}{l}\text { Directeur et } \\
\text { responsable administratif. }\end{array}$ \\
\hline $\begin{array}{l}\text { Cas n }^{\circ} 16 \\
\text { Cendrillon }\end{array}$ & Carrosserie industrielle & $\begin{array}{l}\text { Directeur, } \\
\text { directeur adjoint et } \\
\text { responsable commercial. }\end{array}$ \\
\hline $\begin{array}{l}\text { Cas no } 17 \\
\text { Copévin }\end{array}$ & $\begin{array}{l}\text { Commercialisation } \\
\text { de vins }\end{array}$ & $\begin{array}{l}\text { Directeur, } \\
\text { responsable site et } \\
\text { responsable informatique. }\end{array}$ \\
\hline $\begin{array}{l}\text { Cas no } 18 \\
\text { Coupianum }\end{array}$ & $\begin{array}{l}\text { Machine de découpe à } \\
\text { commandes numériques }\end{array}$ & $\begin{array}{l}\text { Directeur et } \\
\text { responsable commercial. }\end{array}$ \\
\hline $\begin{array}{l}\text { Cas n }{ }^{\circ} 19 \\
\text { Infotek }\end{array}$ & Câblage informatique & $\begin{array}{l}\text { Directeur administratif et } \\
\text { responsable service clients. }\end{array}$ \\
\hline $\begin{array}{l}\text { Cas no } 20 \\
\text { Renaldia }\end{array}$ & $\begin{array}{l}\text { Laboratoire } \\
\text { pharmaceutique }\end{array}$ & $\begin{array}{l}\text { Responsable informatique, } \\
\text { directeur et } \\
\text { responsable commercial. }\end{array}$ \\
\hline $\begin{array}{l}\text { Cas n }^{\circ} 21 \\
\text { Barbarie }\end{array}$ & Maintenance & Directeur \\
\hline
\end{tabular}

\section{Bibliographie}

AKRICH, M., M. CALlON et B. LATOUR (1988), « À quoi tient le succès des innovations », Annales des mines, septembre, p. 14-29.

Alter, N. (1995), «Peut on programmer l'innovation?», Revue française de gestion, avril-mai, p. 78-86.

AMABILE, S., M. GADILLE et R. MEISSONNIER (2000), «Information, organisation, décision : étude empirique sur les rapports des NTIC dans les PME “internautes" », SIM, $\mathrm{n}^{\mathrm{o}} 1$, p. 41-60.

Arthur, B., Y. ERmolieV et Y. KANiOVsKi (1987), «Path dependent processes and the emergence of macro-structure », European Journal of Operational Research, vol. 30 .

Askenazy, P. et C. Gianella (2000), «Le paradoxe de Solow enfin résolu ? », Économie et statistiques, $\mathrm{n}^{\mathrm{0}}$ 339-340 et problèmes écomiques, $\mathrm{n}^{\mathrm{o}} 2720$, p. 20-24.

BAILE, S. (1997), «L'échange de données informatiques. Stratégie interorganisationnelle de la PME », Gestion 2000, juillet, p. 169-184.

BARRAS, R. (1986), « Towards a theory of innovation in services », Research Policy, vol. 15.

Revue internationale P.M.E., vol. 15, nos 3-4, 2002 
BergadaA, M. et B. VidAiLlet (1996), «La décision telle que la voient les décideurs », Revue française de gestion, $\mathrm{n}^{\mathrm{o}} 111, \mathrm{p} .152-158$.

BERGADAA, M. et J. VON BURG (2000), «L'interface entreprise-consommateur : la mutation par les NTIC », Actes du $16^{e}$ congrès international de l'Association française de marketing, R. Michon, J.C. Chebat et F. Colbert (dir.), École des Hautes Études commerciales, Montréal, p. 839-845.

BERGERON, F. et L. RAYMOND (1996), «EDI dans la PME et la grande entreprise : similitudes et différences », Revue internationale PME, vol. 9, nº 1, p. 41-60.

BERgeron, F., L. RAYMOND, M. Gladu et C. LECLERC (1998), «Contribution des technologies de l'information à la performance des PME : quelques dimensions critiques », Actes de la $6^{e}$ Conférence sur les systèmes d'information, Aix-en-Provence.

Boutary, M. (2001), «PME et TIC, les limites de la proximité », Actes du congrès de l'AIM, Nantes, juin.

Bucaille, A. et B. Costa DE BeAuregard (1987), PMI enjeux régionaux et internationaux, Paris, Economica.

BRYNJOLFSSON, E. (1993), «The productivity paradox of information technology : review and assessment », Communications of the ACM, décembre.

BRYNJOLFSSON, E. et L. HitT (1996), «Paradox lost: firm-level evidence on the returns to information system spending », Management Sciences, vol. 42, avril, p. 541-558.

Ciborra, C. (1993), Teams, Market and Systems, Cambridge, Cambridge University Press.

CORIAT, B. et S. GUENNIF (1996), «Incertitude, confiance et institution », Actes du congrès de l'ADSE, la confiance en question, 22 et 23 mars, Aix-en-Provence.

CRÉPON, B. et T. HECKEL (2000), «La contribution de l'informatisation à la croissance française : une mesure à partir des données d'entreprises », Économie et statistiques, $\mathrm{n}^{\circ} 339-340$, p. 93-116.

Croteau, AM., F. Bergeron et L. RAYMond (2001), «Comportements stratégiques, choix et gestion des systèmes d'information, contribution à la performance », Systèmes d'information et management, vol. 6, no 4, p. 5-26.

DURAND, T. (1988), «Management pour la technologie: de la théorie à la pratique », Revue française de gestion, novembre-décembre, p. 5-14.

FABBE-COSTES, N. (2000), «Le rôle transformatif des SIC et TIC sur les interfaces multiacteurs de la distribution et de la logistique », dans Faire de la recherche en distribution et en logistique, FNEGE, Paris, Vuibert, p. 171-193.

FORAY, D. (1989), «Les modèles de compétition technologique, une revue de la littérature », Revue d'économie industrielle, $\mathrm{n}^{\circ} 48$.

GAdille, M. et A. D'IRIBARne (2000), «La diffusion d'internet dans les PME», Réseaux, $n^{\circ} 104$, p. 61-91.

Galliano, D., P. RouX et M. FILIPPI (2000), «Les déterminants organisationnels et spatiaux de l'utilisation des NTIC : le cas des firmes industrielles françaises », Cédérom du $36^{e}$ colloque de l'ASDRLF, septembre, Crans-Montana (Suisse).

Revue internationale P.M.E., vol. 15, ns 3-4, 2002 
GENTHON, CH. et F. ARCANGELI (1997), «Diffusion des nouveaux outils de communication : une comparaison France Italie », $S I M, \mathrm{n}^{0} 3$, p. 7-22.

GIRARD, B. (1997), «Pourquoi les PME restent-elles des PME?», Gérer et Comprendre, $\mathrm{n}^{\circ} 47$, p. 33-42.

GRENAM, N. (1996), «Innovation technologique, changements organisationnels : leur impact sur l'emploi et les qualifications », Économie et statistiques, nº 298, p. 15-34.

HARRISON, D., P. MYKYTYN et RIEMENSCHNEIDER (1997), «Executive decisions about adoption of information technology in small business : theory and empirical tests », Information Science Research, vol. 8, $\mathrm{n}^{\circ}$ 2, p. 171-195.

HLady Rispal, M. (2002), La méthode des cas, Bruxelles, De Boeck Université.

INSEE (2000), « La diffusion des nouvelles technologies de l'information et de la communication dans l'économie », dans Économie française, Comptes de la Nation, Paris, Le Livre de Poche.

JACOB, G. (1993), Le système d' information pour la stratégie d' entreprise, Paris, Hermes.

Julien, P.-A. et M. MARChESNAY (1988), La petite entreprise. Principes économiques et de gestion, Paris, Vuibert.

JULIEN. P.-A., dir. (1994), Les PME : Bilan et perspectives, Paris, Economica; Québec, Les Presses Inter Universitaires.

Julien. P.-A., L. RAYMOND, R. JACOB et C. RAMANGALAHY (1995), « La veille technologique dans les PME manufacturières québécoises », Actes du congrès Innovation et organisation des PME, 25/10, ENST, p. 137-156.

KALIKA, M. (1991), «De l'organisation réactive à l'organisation anticipative », Revue française de gestion, novembre, p. 46-50.

LAROCHE, H. (1995), «Entre décisions et changements stratégiques : vers un modèle de l'action stratégique », Cahier de recherche du groupe ESCP.

LE BAS, C. et A. TORRE (1993), «Survey sur les surveys d'innovation », Revue d'économie industrielle, $\mathrm{n}^{\mathrm{o}} 65$, p. 80-95, $3^{\mathrm{e}}$ trimestre.

Le VigoureuX, F. (1995), «Essai de définition de la moyenne entreprise », Cahiers 1, IAE Basse-Normandie.

MARCh, J.G. (1981), Décision et organisation, Paris, Dunod.

Marchesnay, M. (1992), «Les stratégies de compétitivité des PME», Actes de la conférence "Les PME et la compétivité économique», BFI et OCDE, Montréal, 24-27 mai.

MAYÈRE, A. (2001), « Mutations organisationnelles et évolutions des systèmes et activités d'information et communication », Rapport d'habilitation à diriger des recherches, Université Paul-Sabatier, Toulouse.

MÉRIGOT, J.G. (1991), «Qu'est-ce que l'entreprise?», Travaux de recherche, IAE de Bordeaux.

MONNOYER, M.C. (1990), «Le marché des services vidéotex, une voie pour l'information en ligne», Revue d'économie industrielle, $\mathrm{n}^{\circ}$ 52, p. 39-51.

Revue internationale P.M.E., vol. 15, nos 3-4, 2002 
MONNOYER, M.C. (1997), « Systèmes d'information et productivité », dans M.C. Monnoyer (dir.), L'entreprise et l'outil informationnel, Paris, L'Harmattan.

Rogers, E.M. (1983), Diffusion of Innovations, New York, The Free Press.

SAPORTA, B. (1986), Stratégies pour la P.M.E., Paris, Montchrestien.

SÉRIEYX, H. (1996), Mettez du réseau dans vos pyramides, Paris,Village Mondial.

SESSI, Ministère de l'Industrie (1999), Innovation technologique, Paris, Dunod.

VALLÈs, R.S. et L.V. CARRASCO (1997), «Les PME peuvent-elles obtenir des avantages et bénéfices à partir de l'utilisation d'EDI ? », Gestion 2000, juillet, p. 185-202.

VENKATRAMAN, N. (1991), «IT induced business reconfiguration» dans M.S. ScottMorton (dir.), The Corporate of the 1990s: Information Technology and Organisational Transformation, Oxford, Oxford University Press, p. 122-186.

VenKatraman, N. et J.C. HeNDERSON (1999), «Entreprise virtuelle cherche architecte», Expansion Management Review, mars, p. 6-17. 